\title{
Rectification of Brownian Particles with Oscillating Radii in Asymmetric Corrugated Channels
}

\author{
R. Glaver* AND S. SAVEL'Ev \\ Loughborough University, Loughborough, Leicestershire, LE11 3TU, United Kingdom
}

\begin{abstract}
Transport of a Brownian particle with an oscillating radius freely diffusing in an asymmetric corrugated channel was simulated over a range of driving forces for a series of temperatures and angular frequencies of radial oscillation. It was observed that there was a strong influence of self-oscillation frequency on the average particle velocity. This effect can be used to control rectification of biologically active particles as well as for their separation according to their activity, for instance in the separation of living and dead cells.
\end{abstract}

DOI: 10.12693/APhysPolA.128.159

PACS: 05.40.-a, 87.10.Mn

\section{Introduction}

Rectification is the process of turning the unbiased fluctuations in a system into the directed transport of particles. One means by which this is accomplished is by the use of ratchets. A ratchet is a device that is capable of transporting particles in a periodic structure with non-zero macroscopic velocity, although on average no macroscopic force is acting. This is achieved through the breaking of temporal and/or spatial symmetry [1-4].

Interest in rectification partly stems from its potential application on the nanoscale where Brownian motion predominates, yet it can deliver directed transport of biological probes and nanodevices, as well as, particle separation. However for practical implementation to be achieved, it is apparent that chamber and particle geometry must be considered.

With respect to chamber geometry, it is known that in a variety of biological systems particles move in constrained geometries, such as cavities or channels which act as traps. When particles diffusing between such cavities are surrounded by smooth channels connected by narrow pores they are confined in each cavity by an entropic rather than an energetic potential $[5,6]$. In such cases a Fokker-Planck or Langevin equation can be used to model the diffusion dynamics of these particles in two and three dimensions. At low values of the driving force, approximate solutions can then be obtained by reducing the problem to Brownian diffusion in an effective 1D periodic potential using a Fick-Jacobs kinetic equation with a spatially dependent diffusion function and a 1D entropic barrier replacing the geometric constraints. However, at higher values of the driving force the model parameters of the average particle current and the effective diffusion coefficient diverge from their actual values. This occurs because the assumption of transversely uniform density distribution introduced in the Fick-Jacobs

*corresponding author; e-mail: r.glavey@lboro.ac.uk approximation to eliminate transverse coordinates, is no longer valid at strong driving forces. A general estimate of the criteria under which the Fick-Jacobs equation applies is that $\max \left(\tau_{y} / \tau_{x}, \tau_{y} / \tau_{\text {drift }}\right) \ll 1$, where $\tau_{y}, \tau_{x}, \tau_{\text {drift }}$ are the time scales associated with the diffusion in the transverse direction over distance $\Delta y$, the diffusion in the axial direction and the drift (ballistic) motion respectively [7-9]. Examples of when the 1D reductionist approach has proved inadequate include geometric stochastic resonance (gSR) in a double cavity and Brownian motion in septate channels $[5,6,10,11]$. There are therefore limits to the applicability of the 1D reductionist approach. For sharper boundaries and/or larger driving forces, we must consider alternative approaches such as the Euler algorithm of the Langevin equation in two dimensions as we have used below $[5,6,10,12-16]$. Fortunately, spatial asymmetry is not necessary for rectification, time-asymmetric drives have been numerically and experimentally demonstrated to produce rectification obviating the need for finely constructed channels [17-19].

With respect to particle geometry, we have already demonstrated that in the case of gSR, the elongation aspect ratio $b / a$ of an elliptical particle and its length $b$ relative to pore width $\Delta$ could both be optimised to promote gSR [10]. Furthermore, an elliptical particle with elongation aspect ratio $b / a$, where $a \ll b$, and subject to a direct drive $F_{0}$ and alternating drive $F_{a c}$ diffusing in a highly spatially symmetric channel, has been shown to demonstrate absolute negative mobility (ANM) that could be optimised for drive and particle parameters. This could not be achieved for non-extensive particles [20-22]. These findings have practical implications, as it is much easier to control the fabrication of nanoparticle shape than of the chambers through which they pass.

Interest in particle shape and its effect upon stochastic dynamics is increasing in part due to the potential applications of the Janus particles. These particles are nanoparticles composed of two or more different surfaces whose individual physical and/or chemical properties are distinct and consequently interact differently with the surrounding medium [23]. Their asymmetry of 
design gives the Janus particles unique properties of interaction and a wide variety of potential future applications. These applications include: stabilisation of liquid/liquid and liquid/gas interfaces, nanoprobes [24, 25], biosensors [26], drug delivery, "lab on a chip" devices, tailored substrate wettability [27] and programmable nanostructures capable of self-assembly and reconfiguration to name but a few [23].

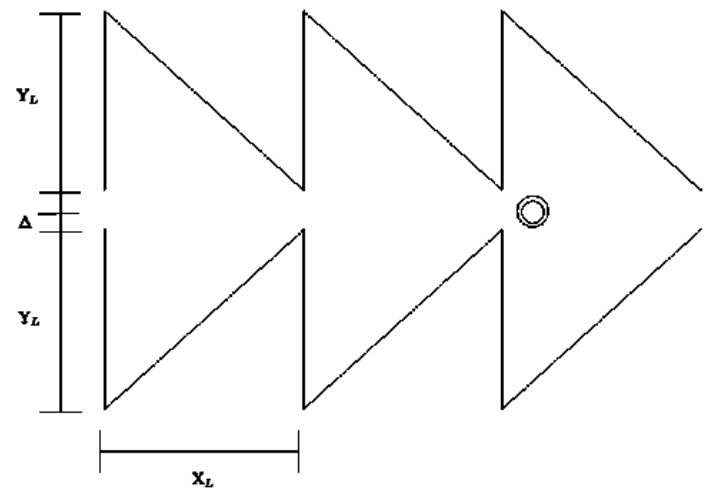

Fig. 1. Brownian particle with oscillating radii freely diffusing in a 2D asymmetric corrugated channel. For Figs. 2 and 3 we used the following channel parameters: $Y_{L}=X_{L}=1, \Delta=0.1$.

Not content with prospects for directed transport on the nanoscale by means of rectification, ANM or gSR however, researchers are investigating the additional possibility of self-propelled Janus particles [23, 28]. One means by which this has been achieved is the catalytic degradation of $\mathrm{H}_{2} \mathrm{O}_{2}$, asymmetrically distributed over the Janus particle surface to release $\mathrm{O}_{2}$ and propel the particle forward [29-33]. However, propulsion by such means is limited by reactant concentrations. An alternative solution might be found at the mesoscopic level where bacteria use various modes of self-propulsion including: flagellar motion, gliding motility, tumbling, screwing and twitching [34-36]. The latter involves the extension of pili from the bacterium followed by their retraction as the bacterium draws itself through the medium. This is not unlike the radial oscillation of the self-propelled Janus-like particles we will describe below. Whatever means of self-propulsion emerges at the nanolevel, it must content with the challenge of Brownian motion and the need for a non-equlibrium source of energy to achieve directed motion [34]. Giant negative mobility of self-propelled elliptical Janus particles in a corrugated channel has already been demonstrated. Numerical simulations have shown that prolate elliptical particles drifted against the axial drive by tumbling over the pore walls for appropriate elongation aspect ratios [37]. With these considerations in mind, we have modelled Janus-like particles in a geometric ratchet composed of an asymmetric corrugated channel, wherein the particles have an element of self-propulsion by means of oscillating radii.

\section{Model and results}

In our model we considered an overdamped Brownian circular particle with an oscillating radius freely diffusing in a 2D suspension fluid confined in an asymmetric corrugated channel with reflecting walls as shown in Fig. 1. The overdamped dynamics of the particles were modelled by the Langevin equation [38, 39]:

$$
\frac{\mathrm{d} \boldsymbol{r}}{\mathrm{d} t}=-A(t) \boldsymbol{e}+\sqrt{D} \boldsymbol{\xi}(t)
$$

where $\boldsymbol{r}$ was the position vector of the particle, $A(t) \boldsymbol{e}$ was the driving force, $\boldsymbol{e}=\left(e_{x}\right)$ was the unit vector, $D$ was the noise intensity and $\boldsymbol{\xi}(t)=\left[\xi_{x}(t), \xi_{y}(t)\right]$ was the zero mean Gaussian white noise with autocorrelation function $\left\langle\xi_{i}(t) \xi_{j}\left(t^{\prime}\right)\right\rangle=2 \delta_{i j} \delta\left(t-t^{\prime}\right)$ with $i, j=x, y$. We used $A(t)=F \operatorname{sgn}\left(\cos \left(\pi t / t_{\max }\right)\right)$ with simulation time $t_{\max }$ and $\operatorname{sgn}(z)=1$ for $z>0$ and $\operatorname{sgn}(z)=-1$ for $z<0$. Thus the ac force $A(t)$ pushed the system to the left during the first half time of simulation and to the right during the second half time of simulation resulting in no average dc drive. Therefore, the average velocities reported in Figs. 2 and 3 below originated from the rectification mechanism. The particle had a fixed internal component of radius $R_{1}=a$ and an oscillating external component of radius $R_{2}=b|\cos \omega(t)|$, where $a$ and $b$ were constants and $\max \left|R_{2}\right|<R_{1}$. Equation (1) was numerically integrated using an Euler algorithm. The number of time steps used was $10^{9}$ (time step was $10^{-4}$ and simulation time was $\left.10^{5}\right)[39,40]$.

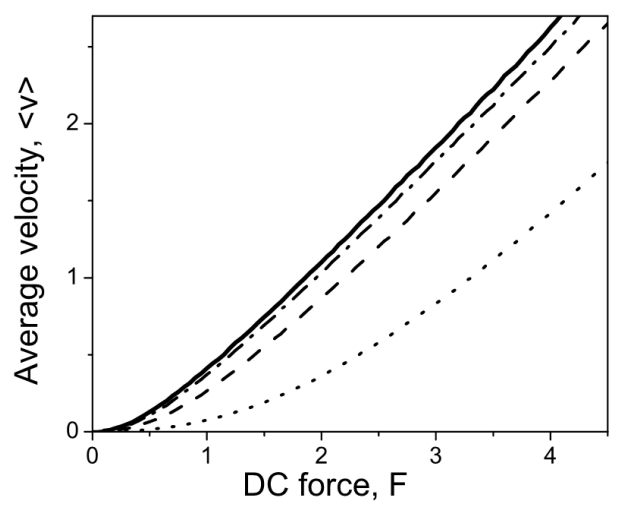

Fig. 2. Average velocity of a single circular particle of constant radius $R(t)=R_{1}\left(R_{2}=0\right)$ as a function of driving force $F$ for several values of $R_{1} / \Delta=0.5$ (solid), 0.8 (dash-dot), 0.95 (dash-dash), 0.99 (dot-dot) with pore size $\Delta=0.1$ (see Fig. 1).

Figure 2 shows rectification of a single circular particle of constant radius $\left(R(t)=R_{1}, R_{2}=0\right)$ for several temperatures and a range of DC driving forces. At low driving forces, the rectified velocity increased as $F^{2}$ and then linearly increased. This behaviour is quite different from the usual rectification peak in 1D. Note that at weak drives rectified velocity can have a more complicated behaviour which is now under investigation and will be reported later. 


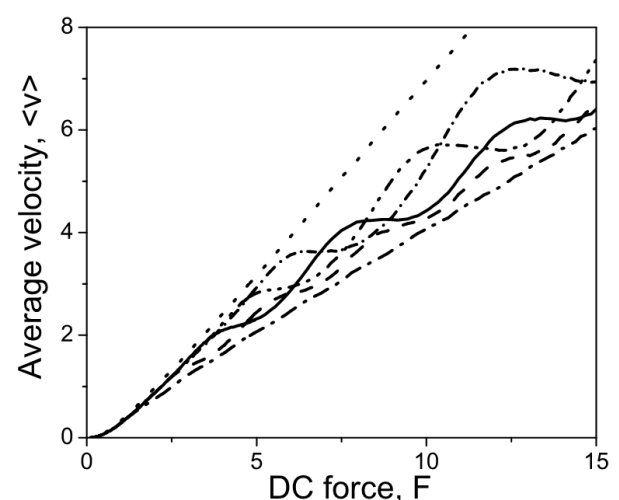

Fig. 3. Average velocity of a self-oscillating particle with an oscillating radius $\left(R_{1}=0.9 \Delta, \max \left|R_{2}\right|=\right.$ $0.3 \Delta)$, diffusion constant $D=0.1$ and a range of oscillation frequencies. The velocity exhibited oscillations with periods controlled by $\omega$.

Figure 3 shows rectification of a self-oscillating particle with a radius composed of a fixed internal component of radius $\left(R_{1}=0.09\right)$ and an oscillating external component of radius $\left(R_{2}=0.03|\cos \omega(t)|\right)$ for fixed temperature $D=0.1$ and a range of angular speeds of oscillation. Thus the particle had a minimum radius of (0.09) and a maximum of $(0.12)$, in a corrugated channel where the pore width was $\Delta=0.10$. This led to a gating mechanism whereby maximal rectification could be achieved when there existed an average time $t_{\mathrm{e}}$ which the particle needed to pass a channel cell (note that this time was controlled by driving amplitude $F$ and noise) and the particle's period of oscillation $t_{\mathrm{o}}$, where $t_{\mathrm{o}}=2 \pi / \omega$ : that is when $t_{\mathrm{e}} \approx n t_{\mathrm{o}}$ and $(n=1,2,3 \ldots)$. The oscillation in each curve for $\omega$ occured because with increased driving force the value of $t_{\mathrm{e}}$ fell successively in and out of commensurability with $t_{\mathrm{o}}$ leading to varying amplitude of rectification. When $t_{\mathrm{o}} \approx t_{\mathrm{e}}$ the particle as "seen" by successive pores most closely resembled a particle of constant radius and thus its rectification most closely resembled that of a particle of constant radius, too. It is apparent then that differential rectification of populations of Brownian particles freely diffusing in asymmetric corrugated channels can be achieved by means of differing angular frequencies of oscillation and used to achieve particle separation. This will be of interest to those seeking to achieve controllable and directed transport of self-oscillating particles on the nanoscale such as that of self-propelled Janus particles.

\section{Conclusion}

We have demonstrated rectification for a self-propelled Brownian particle in a series of asymmetric corrugated channels. Rectification could be optimised for temperature, as expected, and frequency $\omega$ of radial oscillation by matching the mean noise and driving force induced escape time $t_{\mathrm{e}}$ with the period of oscillation $t_{\mathrm{o}}$. This mechanism can be used for separation of self-oscillating particles according to their self-frequencies (for instance living and dead cells or bacteria) on the nanoscale.

\section{Acknowledgments}

S.E.S. was partially supported by Ministry of Science of Montenegro, under Contract No. 01-68.

\section{References}

[1] P. Hänggi, R. Bartussek, in: Nonlinear Physics of Complex Systems: Lecture Notes in Physics, Eds. S.C. Muller, J. Parisi, Springer-Verlag, Berlin 1996, p. 294.

[2] P. Reimann, Phys. Rep. 361, 57 (2002).

[3] R.P. Feynman, R.B. Leighton, M. Sands, in: The Feynman Lectures on Physics, Basic Books, New York 1963, p. 46.

[4] S.J. Lade, Ratchets: Directed transport without a bias force, [Poster]. Exhibited at Australian Institute of Physics 18th National Congress, Adelaide (Australia), 2008.

[5] P.K. Ghosh, F. Marchesoni, S.E. Savel'ev, F. Nori, Phys. Rev. Lett. 104, 020601 (2010).

[6] P.K. Ghosh, R. Glavey, F. Marchesoni, S.E. Savel'ev, F. Nori, Phys. Rev. E 84, 011109 (2011).

[7] P.S. Burada, G. Schmid, D. Reguera, J.M. Rubi, P. Hänggi, Phys. Rev. E 75, 051111 (2007).

[8] R. Zwanzig, J. Phys. Chem. 96, 3926 (1992).

[9] P.S. Burada, P. Hänggi, F. Marchesoni, G. Schmid, P. Talkner, Chem. Phys. Chem. 10, 45 (2009).

[10] M. Read, R. Glavey, F. Marchesoni, S.E. Savel'ev, Europ. J. Phys. B 87, 206 (2014).

[11] M. Borromeo, F. Marchesoni, Chem. Phys. 375, 536 (2010).

[12] F. Marchesoni, S.E. Savel'ev, Phys. Rev. E 80, 011120 (2009).

[13] P.S. Burada, G. Schmid, D. Reguera, M.H. Vanstein, J.M. Rubi, P. Hanggi, Phys. Rev. Lett. 101, 130602 (2008).

[14] M.H. Jacobs, Diffusion Processes, Springer-Verlag, New York 1967.

[15] S. Denisov, S. Flach, P. Hänggi, Phys. Rep. 538, 77 (2014).

[16] P. Hänggi, F. Marchesoni, Rev. Mod. Phys. 81, 387 (2009).

[17] D. Cole, S. Bending, S.E. Savel'ev, A. Grigorenko, T. Tsuyoshi, F. Nori, Nat. Mater. 5, 305 (2006).

[18] S. Denisov, S. Flach, A.A. Ovchinnikov, O. Yevtushenko, Y. Zolotaryuk, Phys. Rev. E 66, 041104 (2002).

[19] S.E. Savel'ev, F. Nori, Nat. Mater. 1, 179 (2002).

[20] P. Hänggi, F. Marchesoni, S.E. Savel'ev, G. Schmid, Phys. Rev. E 82, 041121 (2010).

[21] R. Eichhorn, P. Reimann, P. Hänggi, Phys. Rev. Lett. 88, 190601 (2002).

[22] R. Eichhorn, P. Reimann, P. Hänggi, Phys. Rev. E 66, 066132 (2002). 
[23] A. Walther, A.H.E. Muller, Chem. Rev. 113, 5194 (2013).

[24] J. Choi, Y. Zhao, D. Zhang, S. Chien, Y.H. Lo, Nano Lett. 3, 995 (2003).

[25] J.N. Anker, R. Kopelman, Appl. Phys. Lett. 82, 1102 (2003).

[26] C.J. Behrend, J.N. Anker, B.H. McNaughton, M. Brasuel, M.A. Philbert, R. Kopelman, J. Phys. Chem. B 108, 10408 (2004)

[27] A. Synytska, R. Khanum, L. Ionov, C. Cherif, C. Bellman, Appl. Mater. Interfaces 3, 1216 (2011).

[28] P.K. Gosh, V.R. Misko, F. Marchesoni, F. Nori, Phys. Rev. Lett. 110, 268301 (2013).

[29] S.J. Ebbens, J.R. Howse, Soft Matter 6, 726 (2010).

[30] T-C. Lee, M. Alárcon-Corres, C. Miksch, K. Hahn, J.G. Gibbs, P. Fischer, Nano Lett. 14, 2407 (2014).

[31] J.R. Howes, R.A.L. Jones, A.J. Ryan, T. Gough, R. Vafababakhsh, R. Golestanian, Phys. Rev. Lett. 99, 048102 (2007).
[32] L. Baraban, D. Makarov, R. Streubel, I. Mónch, D. Grimm, S. Sanchez, G. Schmidt, ACS Nano 6, 3383 (2012).

[33] R.A. Pavlick, S. Sengupta, T. McFadden, H. Zhang, A. Sen, Angew. Chem. Int. Ed. 50, 9374 (2011).

[34] R.D. Astumian, P. Hänggi, Phys. Today 55, 33 (2002).

[35] M.J. McBride, Ann. Rev. Microbiol. 55, 49 (2001).

[36] A.J. Merz, M. So, M.P. Sheetz, Nature 407, 98 (2000).

[37] P.K. Ghosh, P. Hänggi, F. Marchesoni, F. Nori, Phys. Rev. E 89, 062115 (2014).

[38] D.S. Lemons, A. Gythiel, Am. J. Phys. 65, 1079 (1997).

[39] Stochastic Processes in Physics and Chemistry, Ed. N.G. Van Kampen, 3rd ed., Elsevier, London 2007.

[40] D.J. Higham, Soc. Industr. Appl. Math. 43, 525 (2001). 